\title{
Numerical Simulation of the Thermomechanical Behavior of a Hot Stamping Die
}

\author{
Q. Y. Jiang, ${ }^{a, 1}$ H. Y Zhao, ${ }^{b}$ and H. F. Yang ${ }^{b}$ \\ ${ }^{a}$ School of Mechanical \& Electrical Engineering, Changchun Institute of Technology, Changchun, \\ China \\ ${ }^{\mathrm{b}}$ School of Materials Science and Engineering, Harbin Institute of Technology (Weihai), Weihai, \\ China \\ 1472699610@qq.com
}

For studying the temperature field of hot stamping, the effect of the distance between cooling pipes, the distance between the cooling pipe and mold surface, and the water cooling pipe diameter were evaluated with ANSYS software. The results showed that the maximum temperature was about $50^{\circ} \mathrm{C}$, and the maximum temperature difference of hot stamping was less than $35^{\circ} \mathrm{C}$. The maximum temperature and the uniformity of stamping temperature can be improved by controlling the size and spacing of cooling pipes.

Keywords: high-strength steel, hot stamping, cooling system, ANSYS, thermomechanical analysis.

Introduction. The application of high-strength steel plate stamping parts in the automobile industry allows to reduce the car body weight with no deterioration of its strength and safety characteristics [1]. Hot stamping technology (HST) is very lucrative for the production of high-strength steel sheet stamping parts, whose strength can exceed $1500 \mathrm{MPa}$, and the formation at high temperature is almost no rebound, with some outstanding advantages of high forming accuracy, good formation, etc. [2-4]. Thus, HST becomes a preferred technology for car manufacturing. Hot stamping die design is the core technology of HST, in which sheet metal forming and quenching are completed in hot stamping die. Therefore, in addition to part-forming requirements, the above die should also possess an excellent cooling capacity to ensure that the parts formed should have good mechanical properties and dimensional accuracy [5-8].

In this paper, the stamping temperature field in high-strength steel hot stamping die was simulated with ANSYS software, with the effect of water cooling pipe diameter, distance between cooling pipes, and the distance between cooling pipes and the mold surface on the temperature distribution being analyzed in detail.

1. Design and Optimization of Cooling System. Mold cooling pipe is the core issue in the designing of hot stamping die. The quality of cooling pipe design directly affects the cooling efficiency of the mold and the cooling uniformity, and directly determines the success of the entire mold design. The design of the cooling pipe mainly includes the determination of the pipeline position parameters and the determination of the pipe diameter and the number of the pipeline. However, there are no exact formulas for the determination of the above parameters, especially for the design of the cooling pipe with a complex mold surface. It is not possible to get the universal design formula.

Schematic diagram of cooling pipe of hot stamping mold is shown in Fig. 1. ANSYS software is used to simulate the cooling process during hot forming. The high strength steel material is $22 \mathrm{MnB} 5$ and the mold material is $\mathrm{CR} 7 \mathrm{~V}$. The influence of distance between the cooling pipe, the distance between the cooling pipe to mold surface, and water cooling pipe diameter on the mold temperature field were analyzed. The differences between the maximum temperature and the maximum temperature of the mold were studied with different mold parameters. 


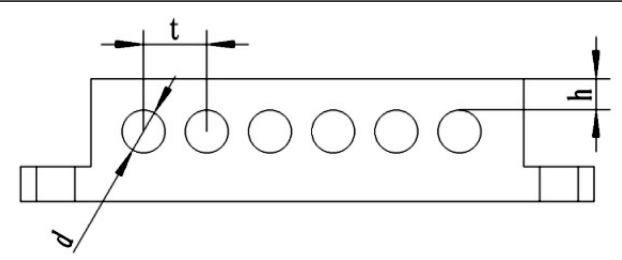

Fig. 1. Schematic diagram of cooling pipe of hot stamping mold.

1.1. The Preliminary Determination to Pipe Position and Size. The location and size of the pipeline mainly depend on the specific shape of the mold. To determine the initial should combine with the design experience. During the design the following points should be paid attention to.

(1) The distance between the cooling pipe and the mold surface and the distance between the cooling pipes should be reduced as small as possible under the condition to meet the strength requirements. This is because that in the mold work the pipeline of horizontal position should withstand more impact and extrusion force. The distance between the cooling pipes is related to the diameter of the pipe. The larger the diameter is, the greater the distance is. Because the strength of the mold depends on its minimum cross-sectional area, the minimum area of the cross section of the pipe must be greater than the allowable critical area.

(2) Pipeline design at rounded corners of convex and concave die. The rounded corners of convex and concave die are subjected to large compressive stress and thermal friction during forming. During the cooling pipe is arranged, the cooling effect is not good with the too far distance of rounded corners and it difficult to ensure the strength of the mold when the distance is too close to make. In the design of the cooling pipe, the die with the force of fillet should maintain a sufficient gap to ensure the strength of the mold, while die (punch) corresponding to the smaller force should reduce the distance between the pipe and mold surface to compensate the other side of the cooling pipe temperature.

1.2. To Check the Strength of the Mold. The lowest value of die mechanical strength is in its smallest cross-section. Drilling in the mold can inevitably lead to lower mold strength. In the design of the cooling pipe, it requires that the minimum cross-sectional area of the mold should be less than the allowable area of the mold. For hot stamping die, due to bear a strong thermal fatigue, the mold safety factor should be higher than the cold forming die.

1.3. To Check the Flow Status of the Pipeline. There are two kinds of cooling water flow in the pipeline: laminar flow and turbulent flow. Only the cooling medium close to the waterway wall in the laminar flow can take away the heat, and the rest of the fluid only flows through the waterway. In the turbulent flow, due to the cooling medium the eddy current is involved in the cooling, the flow of cooling water in the pipeline is required for turbulence flow.

Reynolds number is the dimensionless number to measure the flow of liquid in the water flow. When the Reynolds number is below 2000, the liquid flow state is laminar, and when the Reynolds number is higher than 4000, the transition state is between the two. According to the mold design to determine the initial temperature of the cooling water and parameters of the flow to calculate the Reynolds number in the cooling pipe. The Reynolds number must be greater than 4000 in order to determine if the value of the cooling pipe diameter is qualified.

1.4. To Check Cooling Rate and Cooling Uniformity. The cooling effect check of the cooling system on the mold mainly depends on the numerical simulation, which can not only improve the efficiency of design and development, but also save the cost. Through the analysis to the simulation results, the shortcomings and deficiencies during the design can be found to improve the design. 


\section{Results and Discussion.}

2.1. The Effect of Water Cooling Pipe Diameter. The influence of the diameter of the water pipe on the maximum temperature and the maximum temperature difference of the mold are shown in Fig. 2. It is evident that the maximum temperature of the mold increases as the diameter of the water pipe increases when the other factors of the process do not change. It indicates that with the cooling pipe diameter increases, the cooling rate is slower. At the same time, with the cooling pipe diameter increasing, the maximum temperature difference inside the cooling pipe will increase, which indicates that the smaller the diameter of the pipe, the more even cooling of the mold. It shows that under condition of the same in other parameters, the smaller the diameter of the pipe, the better the cooling effect.

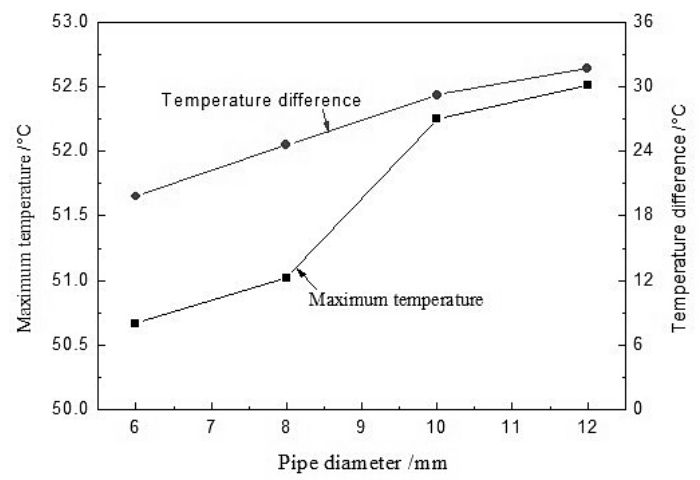

Fig. 2. The influence of water pipe diameter.
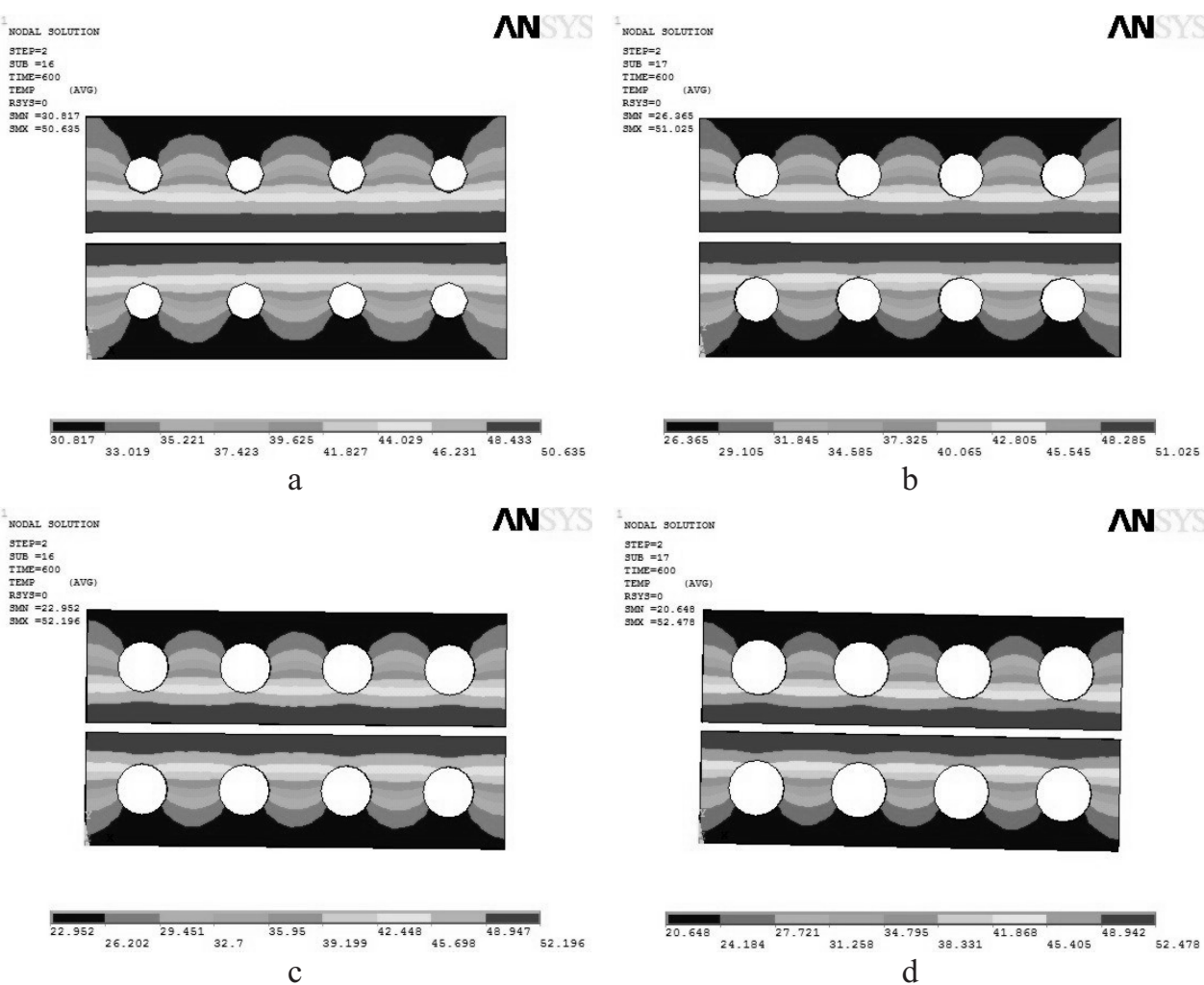

N

Fig. 3. Temperature field of different cooling pipe diameter: (a) $d=6 \mathrm{~mm}$; (b) $d=8 \mathrm{~mm}$; (c) $d=10 \mathrm{~mm}$; (d) $d=12 \mathrm{~mm}$. 
The temperature field for different cooling pipe diameters is shown in Fig. 3. The maximum temperature was observed at the mold surface and did not exceed $55^{\circ} \mathrm{C}$, while the lowest one was observed outside the cooling pipe. With an increase in the cooling pipe diameter, the high-temperature zone dropped gradually and had a wavelike distribution.

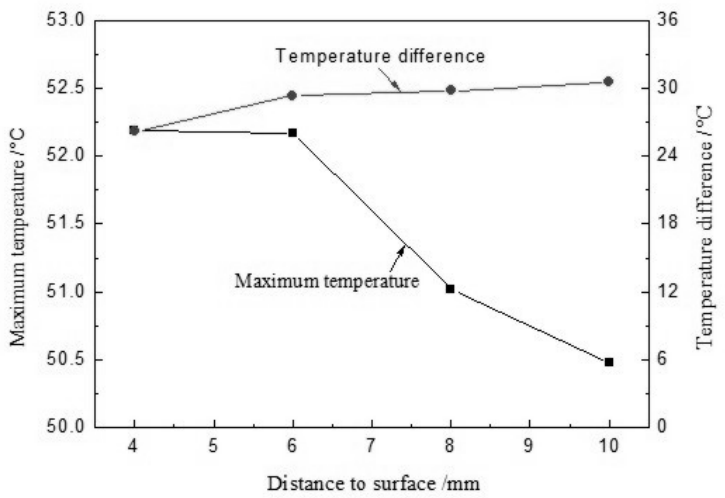

Fig. 4. The influence of distance to mold surface.

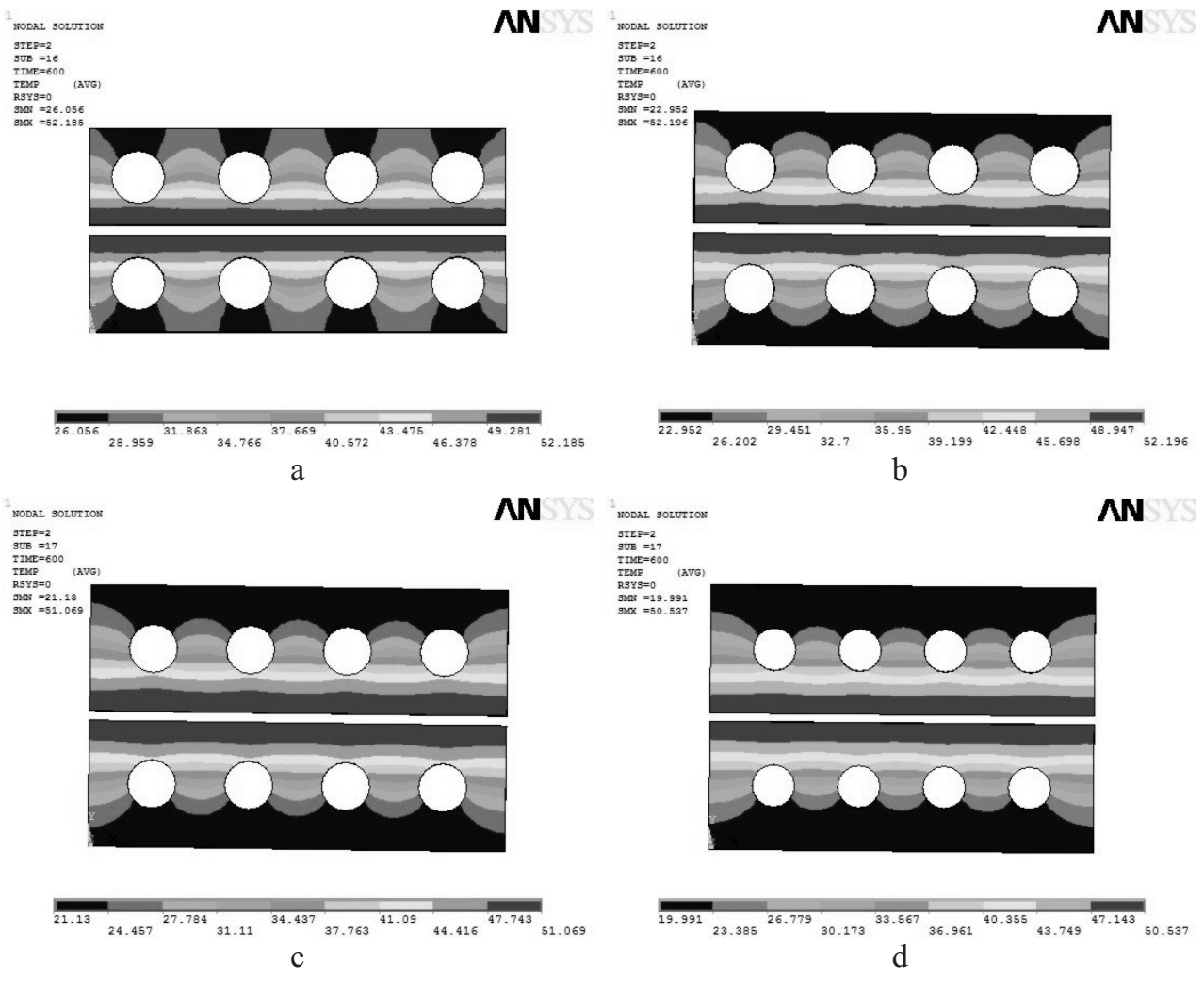

Fig. 5. Temperature field of different distance to mold surface: (a) $h=4 \mathrm{~mm}$; (b) $h=6 \mathrm{~mm}$; (c) $h=8 \mathrm{~mm}$; (d) $h=10 \mathrm{~mm}$.

2.2. The Effect of the Distance between Cooling Pipes and Mold Surface. Figure 4 shows the effect of the wall distance from the water cooling pipe on the maximum temperature and maximum temperature difference. It can be seen that for other conditions 
remaining unchanged, an increase in the distance between the wall and water pipe, the maximum temperature has a downward trend, and the maximum temperature difference shows an upward trend. Therefore, the larger the cavity wall distance of water cooling pipe, the faster the cooling rate.

The temperature field for different distances between the pipe and mold surface is shown in Fig. 5. It can be seen that with an increase in the distance to the mold surface, the high temperature zone between the cooling pipe and the mold surface has a temperature gradient pattern.

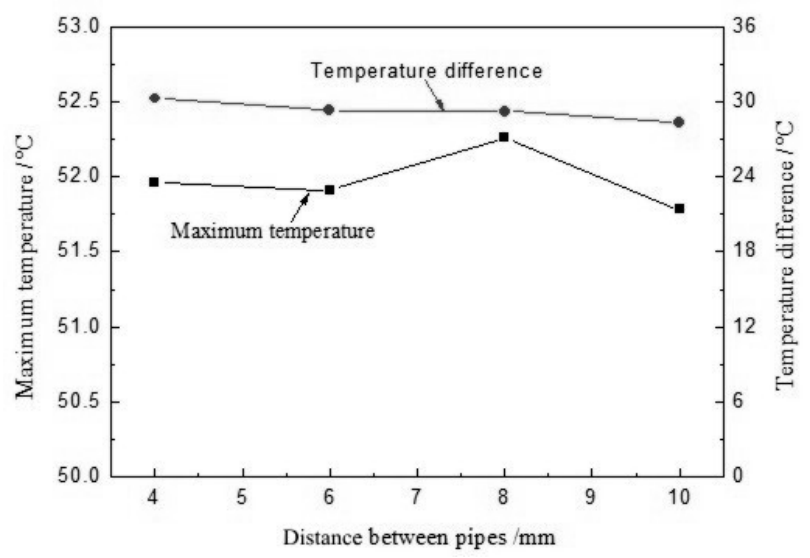

Fig. 6. The influence of distance between pipes.
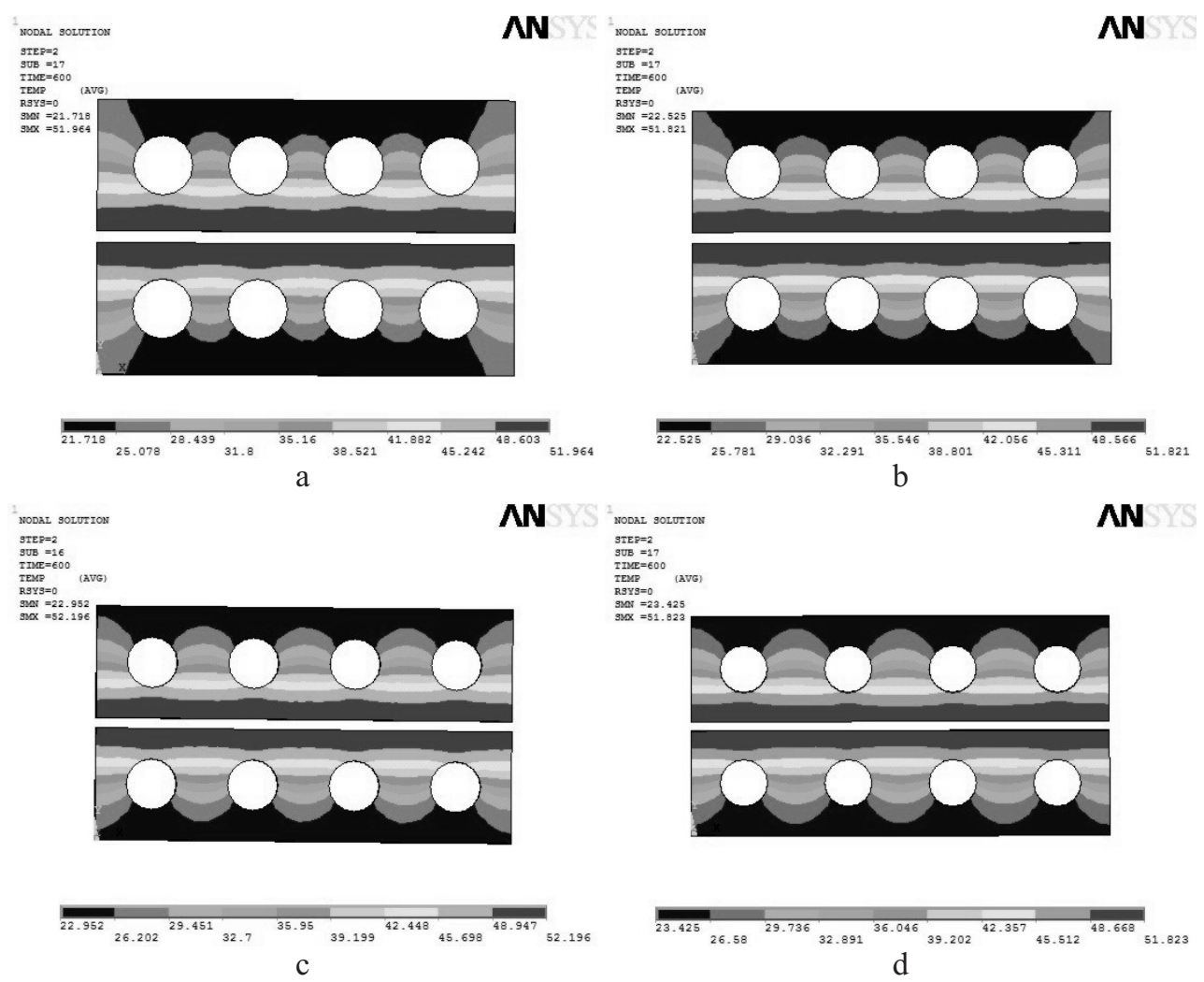

Fig. 7. Temperature field of different distance between pipes: (a) $t=6 \mathrm{~mm}$; (b) $t=8 \mathrm{~mm}$; (c) $t=10 \mathrm{~mm}$; (d) $t=12 \mathrm{~mm}$. 
2.3. The Effect of the Distance between the Cooling Pipes. Figure 6 shows the influence of the space between water cooling pipes on the maximum temperature and the maximum temperature difference of the mold. When the space between water cooling pipes was within a certain range, the maximum temperature fluctuated up and down in a very small range. However, with the increasing spacing between water cooling pipes, the maximum temperature difference of the mold was reduced: the larger the spacing between water cooling pipes, the better the cooling effect.

The temperature field for different distances between pipes is shown in Fig. 7. It can be seen that with an increase in the distance between pipes, the low temperature zone decreases gradually and distributes more unevenly, and the temperature distribution in the high temperature zone slightly varies.

\section{Conclusions}

1. The effect of distance between cooling pipes, the distance between the cooling pipe and mold surface, and the water cooling pipe diameter on the stamping temperature field was analyzed with ANSYS software. The maximum temperature was about $50^{\circ} \mathrm{C}$, and the maximum temperature difference of the hot forming mold was less than $35^{\circ} \mathrm{C}$.

2. The maximum temperature and the uniformity of mold temperature can be improved by controlling the size and spacing of cooling pipes. With an increase in the diameter of water cooling pipes, the maximum temperature increases, and the maximum temperature difference of the mold becomes larger.

3. An increase in the spacing between water cooling pipes results in a sharp reduction of the maximum temperature difference.

Acknowledgments. The project of Jilin Provincial Science and Technology Department "The Study on Ultra-High Gradient Parts Preparation Process and Organizational Performance Based on Zoning Stamping" (No. 20150060). The project of Jilin Province Science and Technology Department "The Research on 22MnB5 Ultra-High Strength Steel Hot Stamping Process" (No. 20140078).

1. Y. Kang, G. Chen, G. Zhu, and R. Song, "Forming technology and application of new generation advanced high strength steel for automobile," Iron Steel, 45, No. 8, 1-6, 19 (2010).

2. L. Fan, "The fatigue analysis and structural optimization to advanced high-strength steel stamping die," J. Hunan Univ. (2014).

3. T. T. Wu, "The forming process analysis and mold development to high-strength steel car cover," J. Shandong Univ. (2015).

4. Z. Zhang, "The hot stamping technology and numerical simulation to high-strength steel plate," Metal Cast. Forg. Technol., 19-23 (2010).

5. X. Wu, "The study on impact of die gap on thermoforming and its and optimization method," J. Hunan Univ. (2015).

6. W. Bi, The Numerical simulation and Application of Hot Stamping Process for Automobile Axle Housing [in Chinese], Doctoral Thesis of Jilin University (2009).

7. R. Wu, "The fatigue life analysis and structural topology optimization to high-strength steel stamping die," J. Hunan Univ. (2015).

8. B. Ding, "The study on fatigue life of ultra-high strength steel plate hot stamping die," J. Chongqing Jiaotong Univ. (2015). 